\title{
EFEITOS DA SUBSTITUIÇÃO PARCIAL DO AÇÚCAR POR SUCRALOSE E GOMA XANTANA SOBRE O PROCESSO DE GELATINIZAÇÃO DO AMIDO EM BOLOS TIPO ESPONJA
}

\author{
A. V. de O. BARBOSA ${ }^{1}$ e C. E. M. da SILVA ${ }^{1}$ \\ 1 Universidade Federal do Ceará, Departamento de Tecnologia de Alimentos \\ E-mail para contato: albavaleriaob@yahoo.com.br
}

\begin{abstract}
RESUMO - O açúcar retarda a gelatinização do amido, devido a sua capacidade de ligarse a água permitindo que o amido atue como um 'cimento, mantendo as células de ar distribuídas e fixadas à estrutura no processo final de assamento, caracterizando a formação porosa do bolo esponja. A redução do açúcar pode vir a afetar as temperaturas de gelatinização do amido, prejudicando a formação estrutural dos bolos. Objetivou-se analisar o efeito da substituição parcial do açúcar por sucralose e goma xantana nas temperaturas de gelatinização do amido de massas do bolo esponja, comparando os resultados com uma amostra controle. Valores de To $60-88,5^{\circ} \mathrm{C}$ e Tp $99,3-110,5^{\circ} \mathrm{C}$ de gelatinização foram superiores ao controle, esse aumento da temperatura retardou acima do esperado a gelatinização permitindo o escape do ar, devido a redução da viscosidade, resultando em bolos de menor volume. Valores de $\mathrm{Tf} 127,2-121,1^{\circ} \mathrm{C}$ inferiores ao controle, influindo na $\Delta \mathrm{T} 66,4-35,3$ que foram inversamente proporcionais aos de $\Delta \mathrm{H}$ $138,3-747,8 \mathrm{~J} / \mathrm{g}$ sugerindo que houve um estreitamento da faixa de gelatinização, associado a altos valores de $\Delta \mathrm{H}$ são um indicativo do enrijecimento da estrutura do bolo, impedindo a expansão do ar ocasionando a redução do volume, confirmado através do volume especifico $1,90-1,05 \mathrm{~cm}^{3} / \mathrm{g}$, que diminuíram ao passo que o açúcar foi substituído. Conclui-se que a substituição parcial do açúcar por sucralose e goma xantana afetou negativamente a gelatinização do amido resultando em bolos de menor volume.
\end{abstract}

\section{INTRODUÇÃO}

Alimentos com conteúdo reduzido de açúcar vem ganhando cada vez mais popularidade devido o desejo da população em reduzir calorias na sua dieta, que além de prevenir o ganho de peso, evita o surgimento problemas na saúde como hipertensão e doenças cardiovasculares que geralmente são associados a doenças crônicas como a obesidade e o diabetes Psimouli e Oreopoulou (2012).

Bolos tipo esponja, são apreciados em todo mundo e por conter altas proporções de açúcar, são candidatos ideais de pesquisas que almejam o desenvolvimento de produtos com reduzido teor calórico Kocer et al. (2007), substituindo o açúcar por um adoçante de alta intensidade e um espessante de baixa caloria Bennion e Bamford (1997), pois nenhum agente espessante possui todas as propriedades únicas conferidas ao açúcar. 


\section{9 a 22 de outubro de 2014 \\ Florianópolis/SC}

A sucralose é um adoçante sintetizado a partir da sacarose em que três grupos hidroxilas, nas posições 4, 1 e 6 foram substituídos por 3 átomos de cloro resultando no composto 1,6-dicloro-1,6 dideoxi- $\beta$-D-fructofuranosil-4-Cloro-4-deoxi- $\alpha$-D-galactopiranose Rodero et al. (2009), com poder adoçante 600 vezes superior a sacarose (Barndt e Uackson, 1990; Tozetto, 2005) não apresenta sabor residual amargo característico de outros adoçantes como a sacarina, por ser estável a altas temperaturas e a acidez elevada, mantendo sua doçura durante o cozimento, seu uso como substituto do açúcar é recomendado em produtos de panificação, porém apenas como agente de doçura, embora seja sintetizada a partir da sacarose, a sucralose não possui a capacidade apresentada por essa, de contribuir para a formação estrutural de produtos de panificação como os bolos (Lin e Lee, 2005; Martinez-Cevera et al., 2012), por isso que a combinação de agentes de volume deve ser usado como alternativa para a melhoria das características estruturais dos bolos Baeva et al. (2000).

Pois o açúcar exerce papel fundamental na formação estrutural dos bolos, pois rege todos os fenômenos relacionados como, o retardamento da gelatinização do amido, devido a sua capacidade de ligar-se a agua, formando pontes entre as cadeias de amido, agindo como agente de retenção de umidade, controlando também a desnaturação proteica que começam a uma temperatura crítica mínima e procedem apenas na presença de umidade suficiente (Baeva et al., 2003; Cavalcante, 2012). As proteínas desnaturadas são responsáveis pela armação estrutural da massa, com o atraso da gelatinização do amido este atua como agente selante, mantendo as células de ar bem distribuídas e fixadas a estrutura, fortalecendo-a, é o responsável pela formação porosa característica dos bolos tipo esponja (Kocer et al., 2007; Mizukosh et al., 1980; Schimer et al., 2012). Sob essa perspectiva esse trabalho tem como objetivo analisar o efeito da substituição crescente do açúcar por sucralose e goma xantana nas temperaturas de gelatinização do amido nas massas de bolo esponja, comparando os resultados com uma amostra controle.

\section{MATERIAL E MÉTODOS}

\section{1-Material}

Para o preparo do bolo controle, usado para fins comparativos utilizou-se ingredientes convencionais adquiridos, no comércio de Fortaleza: Farinha de trigo Tipo 1 sem fermento, açúcar cristal, margarina sem sal, ovos tamanho médio de cor branca, leite em pó integral, fermento químico em pó e água mineral.

No preparo dos bolos com reduzido teor calórico, além dos ingredientes convencionais utilizou-se também o adoçante (sucralose), utilizado como agente de doçura, que foi cedido gentilmente pela TATE \& LYLE (São Paulo, Brasil) e o agente de volume (goma xantana) foi fornecido pela Gastronomy lab (Brasília-DF).

\section{3-Métodos}

Preparo dos bolos Para o preparo dos bolos com teor calórico reduzido, utilizou-se uma formulação controle otimizada por Cavalcante (2012) apresentada na tabela 01, Foram elaboradas 7 (sete) formulações, mantendo fixas as proporções dos ingredientes, farinha de trigo, leite em pó, 
fermento químico, margarina, variando apenas a quantidade de açúcar no qual foi substituído em reduções crescentes por uma solução conjunta de sucralose a 1\%, e goma xantana a 1,5\%, totalizando 7 formulações, cuja codificação baseou-se no nível de substituição do açúcar pela solução de sucralose e goma xantana de acordo com o exposto na tabela 02, sendo F0, a formulação controle contendo apenas o açúcar na formulação.

Tabela 01- Formulação de bolo tipo esponja otimizada

\begin{tabular}{cc}
\hline Ingredientes & Gramas \\
\hline Farinha & 100 \\
Açúcar & 155,88 \\
Margarina & 28,78 \\
Leite em pó & 6,79 \\
Ovos & 46,96 \\
Fermento químico & 1,00 \\
Água & 59,59 \\
\hline
\end{tabular}

Tabela 02- Formulações de bolos preparadas com substituição do açúcar pela solução conjunta de Sucralose e goma xantana

\begin{tabular}{cccc}
\hline $\begin{array}{c}\text { Codificação } \\
\text { das } \\
\text { formulações }\end{array}$ & $\begin{array}{c}\text { Nível de substituição } \\
\text { do açúcar pela solução } \\
\text { adoçante e goma }(\%)\end{array}$ & $\begin{array}{c}\text { Proporção de } \\
\text { açúcar } \\
(\mathrm{g})\end{array}$ & $\begin{array}{c}\text { Proporção de solução } \\
\text { de adoçante e goma } \\
(\mathrm{g})\end{array}$ \\
\hline Controle (F0) & 0,00 & 155,88 & 0,00 \\
F10 & 10,00 & 140,28 & 31,17 \\
F19 & 19,00 & 126,27 & 59,23 \\
F27 & 27,10 & 113,64 & 84,49 \\
F34 & 34,39 & 102,28 & 107,21 \\
F40 & 40,95 & 92,05 & 127,66 \\
F46 & 46,86 & 82,83 & 146,09 \\
F52 & 52,17 & 74,56 & 162,64 \\
\hline
\end{tabular}

Definida os formulações, procedeu-se o preparo dos bolos, onde utilizou-se o método rápido de preparo no qual consiste em adicionar e bater os ingredientes em uma só etapa sem acarretar prejuízos a formação estrutural do bolo, depois de misturados os ingredientes foram batidos em batedeira comum com 3 velocidades por 1 (um) min. na velocidade 1, para permitir a incorporação de ar necessária para o desenvolvimento dos bolos, depois de batidos os ingredientes, a massa obtida foi 


\section{9 a 22 de outubro de 2014 \\ Florianópolis/SC}

vertida em forma tipo $\mathrm{W}$ e levada para assar em forno elétrico previamente aquecido por 30 mins. a $180^{\circ} \mathrm{C}$, a massa foi assada a mesma temperatura por aproximadamente 40 mins. e resfriadas a temperatura ambiente e armazenados em recipientes plásticos sob refrigeração. Antes de serem levadas para o assamento, amostras das massas foram retiradas inclusive da formulação controle, realizando-se posteriormente a analise térmica (gelatinização do amido), depois de assados efetuou-se a medição do volume especifico da fatia dos bolos.

Análise das propriedades térmicas (gelatinização do amido) da massa do bolo tipo esponja Segundo metodologia adotada por Wilderjans $e t$ al. (2008). Para a obtenção das propriedades térmicas da massa, as amostras foram analisadas em um calorímetro diferencial exploratório (DSC) modelo 200F3 da Netzsch, com amostras de até 8,0 mg das massas dos bolos com redução de açúcar, em cadinhos de alumínio selados, tendo como referência um cadinho de alumínio selado vazio, com uma velocidade de aquecimento de $10^{\circ} \mathrm{C} / \mathrm{min}$ no intervalo de temperatura de $20-250^{\circ} \mathrm{C}$. As características da gelatinização do amido nas massas de bolo em um termograma de DSC foi indicada por diferentes temperaturas: To (temperatura inicial de gelatinização) e Tf (Temperatura final de gelatinização), Tp (Temperatura de pico, Temperatura de fluxo máximo de calor diferencial), $\Delta \mathrm{T}$ (obtida pela diferença entre a temperatura final e a temperatura inicial) representa a faixa de temperatura de gelatinização e entalpia $\Delta \mathrm{H}$ (quantidade de energia necessária para completar o processo de gelatinização). Análise feita com três repetições.

Volume especifico dos bolos Depois de assado os bolos, efetuou-se o quarteamento para melhor representatividade da amostra, efetuando em seguida a medição do volume especifico da fatia dos bolos segundo metodologia descrita pela AACC (2000), por deslocamento da semente de painço. Análise feita em triplicata.

Tratamento estatístico Os resultados obtidos com as análises térmicas da massa dos bolos e o volume especifico dos bolos, foram submetidas à análise estatística, para tanto, usou-se o Delineamento Inteiramente Casualizado (DIC), onde foram feitas análises de variância (ANOVA) e cálculo do desvio das médias com posterior comparação das diferenças entre as médias pelo teste de Tukey a um nível de significância de $p<0,05$, utilizando o programa estatístico Statistica versão 8 .

\section{0-RESULTADO E DISCUSSÃO}

As medidas de temperatura de gelatinização das amostras de massas de bolo (To, Tp, Tf) com substituição parcial do açúcar por sucralose como substituto do açúcar, de acordo com a tabela 03 diferiram significativamente entre si, para valores de To $\left(60,6-88,5^{\circ} \mathrm{C}\right)$ e $\mathrm{Tp}\left(105,6-110,1^{\circ} \mathrm{C}\right)$, superiores a controle $\left(63,6^{\circ} \mathrm{C}\right)$, no entanto valores de $\mathrm{Tf}\left(127,2-124^{\circ} \mathrm{C}\right)$, foram inferiores ou igual ( no caso da F27) ao controle $\left(126,8^{\circ} \mathrm{C}\right)$ com exceção das formulações $\mathrm{F} 10$ e $\mathrm{F} 19$, que apresentaram valores de To, Tp e Tf inferiores a controle. 
Tabela 03-Temperaturas inicial (To), Temperatura de pico (Tp) e Temperatura final (Tf) de gelatinização do amido em massas de bolo com substituição parcial do açúcar por sucralose e goma xantana

\begin{tabular}{cccc}
\hline Formulações & To $\left({ }^{\circ} \mathrm{C}\right)$ & $\mathrm{Tp}\left({ }^{\circ} \mathrm{C}\right)$ & $\mathrm{Tf}\left({ }^{\circ} \mathrm{C}\right)$ \\
\hline F0 & $63,6^{\mathrm{f}} \pm 0,21$ & $101,7^{\mathrm{e}} \pm 0,14$ & $126,8^{\mathrm{c}} \pm 0,08$ \\
F10 & $60,6^{\mathrm{g}} \pm 0,14$ & $99,3^{\mathrm{f}} \pm 0,18$ & $127,2^{\mathrm{b}} \pm 0,07$ \\
F19 & $57,3^{\mathrm{h}} \pm 0,11$ & $94,3^{\mathrm{h}} \pm 0,15$ & $128,9^{\mathrm{a}} \pm 0,14$ \\
F27 & $76,2^{\mathrm{c}} \pm 0,12$ & $109,8^{\mathrm{b}} \pm 0,18$ & $126,4^{\mathrm{c}} \pm 0,14$ \\
F34 & $74,3^{\mathrm{d}} \pm 0,11$ & $102,9^{\mathrm{d}} \pm 0,11$ & $124,7^{\mathrm{d}} \pm 0,18$ \\
F40 & $82,2^{\mathrm{b}} \pm 0,11$ & $107,8^{\mathrm{c}} \pm 0,25$ & $121,1^{\mathrm{f}} \pm 0,07$ \\
F46 & $73,4^{\mathrm{e}} \pm 0,22$ & $98,7^{\mathrm{g}} \pm 0,12$ & $123^{\mathrm{e}} \pm 0,07$ \\
F52 & $88,5^{\mathrm{a}} \pm 0,14$ & $110,5^{\mathrm{a}} \pm 0,18$ & $124^{\mathrm{d}} \pm 0,07$ \\
\hline
\end{tabular}

Resultados apresentados como valores médios \pm desvio padrão, seguidos por letras sobrescritas diferentes na mesma coluna são significativamente diferentes $(P<0,05)$ onde $a>b>c>d>e>f>g>h$

A figura 01 apresenta os termogramas das curvas de fluxo de calor (faixa de gelatinização do amido) das massas de bolo com substituição parcial do açúcar por sucralose e goma xantana, onde é possível visualizar, o efeito do uso da solução de sucralose sobre a To, Tp e Tf de gelatinização ao passo que efetua-se a substituição crescente do açúcar pela solução de adoçante em questão, sendo F0 a controle:

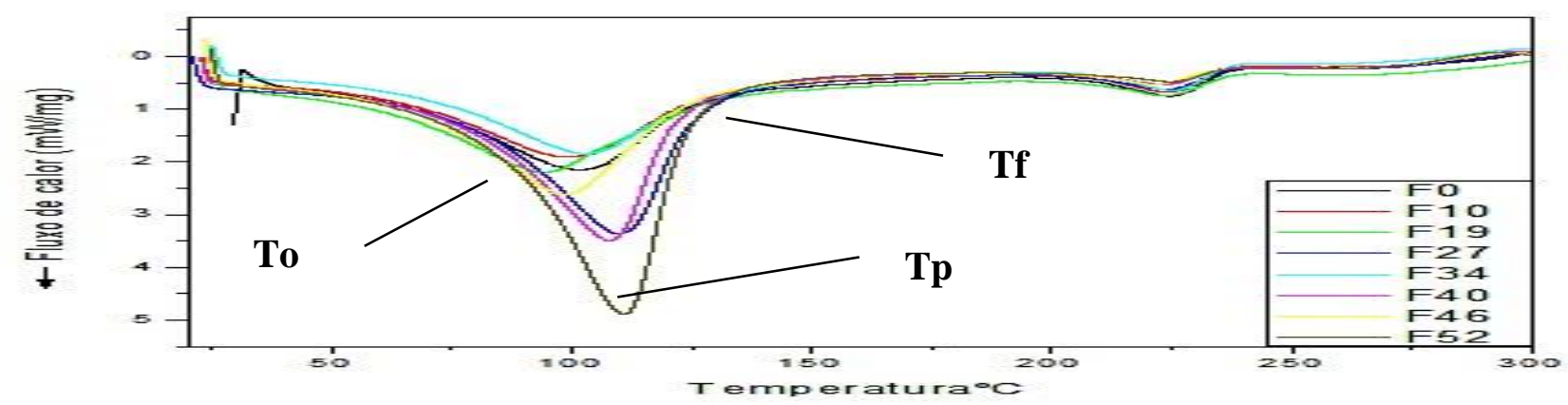

Figura-01-: Faixa de temperatura de gelatinização do amido da massa do bolo com substituição parcial do açúcar por uma solução de sucralose e goma xantana.

A elevação da Tp e o declínio da Tf de gelatinização, influíram negativamente nos resultados de $\Delta \mathrm{T}$, de acordo com a tabela 04 , diferindo significativamente entre si, variando de $71,3-35,3^{\circ} \mathrm{C}$, com valores inferiores ao controle $\left(63,2^{\circ} \mathrm{C}\right)$, indicando que ouve um estreitamento da faixa de gelatinização do amido, conforme o açúcar foi substituído pela solução de sucralose e goma xantana. Esses valores de $\Delta \mathrm{T}$ foram inversamente proporcionais aos de $\Delta \mathrm{H}$ no qual diferiram significativamente entre si, variando de $138-747 \mathrm{~J} / \mathrm{g}$, resultados esses superiores ao controle $(180 \mathrm{~J} / \mathrm{g})$, onde verificou-se o aumento da $\Delta \mathrm{H}$ conforme efetuava-se a substituição crescente do açúcar pela solução de sucralose e goma xantana, com exceção das formulações F10 e F19 que apresentaram 
valores de $\Delta H$ inferiores e $\Delta \mathrm{T}$ superiores ao controle. Segundo Gaonkar e Mcpherson (2005) todos os adoçantes têm a capacidade de influenciar o ponto de gelatinização do amido, elevando a temperatura de gelatinização, esse fato é atribuído à competição dos adoçantes pela agua presente, já que os mesmos possuem afinidade maior que o amido e consequentemente, há menos água disponível para hidratar e gelatinizar o amido, alterando o ponto de gelatinização. Em produtos de panificação como bolos, podem ser negativamente afetados, como redução do volume e estabilidade dos mesmos.

A entalpia $(\Delta \mathrm{H})$ refere-se à quantidade de energia gasta para completar o processo de gelatinização do amido que é endotérmico, ou seja, as formulações com níveis maiores de substituição do açúcar pelo adoçante em questão, necessitaram de uma maior quantidade de energia para completar o processo de gelatinização do amido. Sugerindo que a redução da disponibilidade de água do sistema pela presença da sucralose resultou em um aumento da energia requerida para a gelatinização e consequentemente, temperaturas maiores foram requeridas para dar início a esse processo, afetando negativamente a faixa de gelatinização do amido.

A elevação na entalpia de gelatinização do amido foi associado por Psimouli e Oreopoulou (2012) ao enrijecimento da estrutura da massa, suprimindo o desenvolvimento das células de ar, aumentando a pressão de vapor interna ocasionando a coalescência das células limitando a expansão da massa dos bolos. Portanto a elevação da temperatura de gelatinização, e consequentemente o aumento do $\Delta \mathrm{H}$, proporcionou o estreitamento da faixa de gelatinização, ou seja o amido não gelatinizou adequadamente, limitando assim a expansão da massa, já prejudicada pela redução brusca da viscosidade, prejudicando o volume dos bolos, como apresentado na tabela 04, os volumes específicos dos bolos variaram de $1,90-1,06 \mathrm{~cm}^{3} / \mathrm{g}$, em relação ao controle $\left(1,81 \mathrm{~cm}^{3} / \mathrm{g}\right)$, teve um decréscimo a partir da formulação F27.

Tabela 04- $\Delta \mathrm{T}$ e $\Delta \mathrm{H}$ de gelatinização do amido de massas e volume especifico dos bolos com substituição parcial do açúcar por uma solução de sucralose e goma xantana

\begin{tabular}{cccc}
\hline Formulações & $\begin{array}{c}\Delta \mathrm{T}(\mathrm{Tf}-\mathrm{To}) \\
\left({ }^{\circ} \mathrm{C}\right)\end{array}$ & $\begin{array}{c}\Delta \mathrm{H} \\
(\mathrm{J} / \mathrm{h})\end{array}$ & $\begin{array}{c}\text { Volume especifico } \\
\left(\mathrm{cm}^{3} / \mathrm{g}\right)\end{array}$ \\
\hline F0 & $63,2^{\mathrm{c}} \pm 0,20$ & $180^{\mathrm{e}} \pm 0,70$ & $1,81^{\mathrm{a}} \pm 0,01$ \\
F10 & $66,4^{\mathrm{b}} \pm 0,26$ & $138^{\mathrm{g}} \pm 1,00$ & $1,90^{\mathrm{a}} \pm 0,02$ \\
F19 & $71,3^{\mathrm{a}} \pm 0,20$ & $129^{\mathrm{h}} \pm 1,08$ & $1,80^{\mathrm{a}} \pm 0,06$ \\
F27 & $50,3^{\mathrm{d}} \pm 0,1$ & $177^{\mathrm{f}} \pm 0,70$ & $1,50^{\mathrm{b}} \pm 0,01$ \\
F34 & $50,4^{\mathrm{d}} \pm 0,30$ & $298,7^{\mathrm{d}} \pm 0,11$ & $1,29^{\mathrm{c}} \pm 0,02$ \\
F40 & $38,7^{\mathrm{f}} \pm 0,2$ & $505,1^{\mathrm{b}} \pm 0,07$ & $1,19^{\mathrm{cd}} \pm 0,02$ \\
F46 & $49,4^{\mathrm{e}} \pm 0,20$ & $410,9^{\mathrm{c}} \pm 0,12$ & $1,10^{\mathrm{d}} \pm 0,03$ \\
F52 & $35,3^{\mathrm{g}} \pm 0,2$ & $747,8^{\mathrm{a}} \pm 0,15$ & $1,06^{\mathrm{d}} \pm 0,03$ \\
\hline
\end{tabular}

Resultados apresentados como valores médios \pm desvio padrão, seguidos por letras sobrescritas diferentes na mesma coluna são significativamente diferentes $(P<0,05)$ onde $a>b>c>d>e>f>g>h$ 


\section{9 a 22 de outubro de 2014 \\ Florianópolis/SC}

Durante o aquecimento a viscosidade da massa do bolo, diminui o que é desejável, pois permite que as células de ar provenientes da mistura dos ingredientes e da fermentação química na qual libera $\mathrm{CO}_{2}$, se expandam, quando inicia-se a gelatinização do amido controlada pela presença do açúcar, ocorre o aumento da viscosidade da massa, que impede o escape das células, retendo-as e aprisionando-as, formando assim a estrutura porosa com o volume desejado.

Em estudo realizado por Lin e Lee (2005) a substituição gradual do açúcar por dextrina e sucralose provocou a elevação da temperatura de gelatinização, resultando na diminuição da viscosidade da massa durante o assamento, reduzindo a capacidade de retenção de ar, produzindo bolos com um menor volume indesejável. O aumento demasiado da temperatura, retardou acima do desejado a gelatinização do amido permitindo assim que as células de ar escapem, devido a redução da viscosidade, resultando em bolos de volume reduzido (Psimouli e Oreopoulou, 2012; Ronda et al., 2011). As formulações F10 e F19 apresentaram resultados menores, porém semelhantes ao controle sugerindo que os efeitos advindos da substituição do açúcar pela solução de sucralose e goma xantana sobre a gelatinização do amido que acarretou em prejuízos diretos a expansão e consequentemente o volume desejado dos bolos, iniciou-se efetivamente a partir da formulação F27.

\section{0-CONCLUSÃO}

Conclui-se que, a substituição do açúcar pela solução de sucralose e goma xantana, afetou negativamente a gelatinização do amido das massas de bolo tipo esponja, acarretando em prejuízos a sua estrutura permitindo o escape de parte das células de ar durante o assamento e limitando a expansão das mesmas devido ao enrijecimento da estrutura da massa, resultando em bolos de baixo volume.

\section{0- REFERÊNCIAS}

AACC International. Approved methods of the AACC. $10^{\text {th }}$ ed. AACC The Association: St Paul, Minnesotta, 2000.

BAEVA, M. R.; PANCHEV, I. N.; TERZIEVA, V. V. Comparative study of texture of normal and energy reduced sponge cakes. Narhung, v. 44, p. 242-246, 2000.

BAEVA, M. R.; TERZIEVA, V. V.; PANCHEV, I. N. Structural development of sucrosesweetened and sucrose-free sponge cakes during baking. Narhung, v.47, p. 154-160, 2003.

BARNDT, R.L.; JACKSON G. Stability of sucralose in baked goods, Food Technol., V.44, p. 62-66, 1990.

BENNION, E.B.; BAMFORD, G. S. T. The Technology of Cake Making. London: Blackie Academic e Professional, 1997.

CAVALCANTE, R. S. Avaliação das características estruturais de bolos com redução calórica. Dissertação (Mestrado em Ciência e Tecnologia de Alimentos). Universidade Federal do Ceará, Fortaleza, 2012. 
GAONKAR, A. G.; McPHERSON, A. Ingredient interactions: Effects on food quality. Taylor \&Francis, 2005.

LIN, S-D.; LEE, C-C. Qualities of chiffon cake prepared with indigestible dextrin and sucralose as replacement for sucrose. Cereal Chem., v. 82, p. 405-413, 2005.

KOCER, D.; HICSASMAZ, Z.; BAYINDIRLI, A.; KATNAS, S. Bubble and pore formation of the high-ratio cake formulation with polydextrose as a sugar- and fat-replacer. J. Food Eng., v. 78, p. 953-964, 2007.

MARTÍNEZ-CERVERA, S.; SANZ, T.; FISZMAN, SM. Rheological, textural and sensorial properties of low-sucrose muffins reformulated with sucralose/polydextrose. LWT - Food Sci. Technol., V. 45, n. 2, p. 213-220, 2012.

MIZUKOSHI, M., KAWADA, T., MATSUI, N. Model studies of cake baking. II Expansion and heat set of cake bater during baking. Cereal Chem., v. 57, p. 352-355, 1980.

PSIMOULI, V.; OREOPOULOU, V. The effect of alternative sweeteners on batter rheology and cake properties. J. sci. Food Agric., v. 92, p. 99-105, 2012.

RONDA, F.; OLIETE, B. GOMEZ, CABALLERO, P. A., PANDO, V. Rheological study of layer cake batters made with soybean protein isolate and different starch sources. J. Food Eng., v. 102, p.272-277, 2011.

RODERO, A. B., RODERO, L. S., AZOUBEL, R. Toxicity of Sucralose in Humans: A Review. Int. J. Morphol., v. 27, p. 239-244, 2009.

SCHIRMER, M.; JEKLEA, M.; ARENDTB, E.; BECKER, T. Physicochemical interactions of polydextrose for sucrose replacement in pound cake. Food Res. Int., V. 49, p. 291-298, 2012.

TOZETTO, A. Controle de qualidade de edulcorantes em adoçantes comerciais via espectrometria e métodos de calibração multivariada. Dissertação (Mestrado em ciência e Tecnologia de Alimentos) Universidade Federal de Ponta Grossa, Ponta Grossa, 2005.

WILDERJANS, E.; PAREYT, B.;GOESAERT, H.; BRIJS, K.; DELCOUR, J. A. The role of gluten in a pound cake system: A model approach based on gluten-starch blends. Food Chem., v. 110, p. 909-915, 2008. 